\title{
Effect of Clenching on Spinal Alignment in Normal Adults
}

\author{
Mutsumi Takahashi, ${ }^{1, *}$, Yogetsu Bando ${ }^{2}$, Takuya Fukui ${ }^{3}$, Akiko Maruyama ${ }^{3}$, and Masaaki Sugita ${ }^{4}$ \\ ${ }^{1}$ Department of Physiology, The Nippon Dental University School of Life Dentistry at Niigata, Japan \\ ${ }^{2}$ Bando Dental Clinic, Ishikawa, Japan \\ ${ }^{3}$ Department of Sport Science, Kanazawa Gakuin University of Sport Science, Ishikawa, Japan \\ ${ }^{4}$ Faculty of Sport Science, Nippon Sport Science University, Tokyo, Japan
}

*Corresponding author: Mutsumi Takahashi, Department of Physiology, The Nippon Dental University School of Life Dentistry at Niigata, 1-8, Hamaura-cho, Chuo-ku, Niigata 951-8580, Japan, Tel: +81-25-267-1500; E-mail: mutsumit@ngt.ndu.ac.jp

Received: 10 Dec, 2021 | Accepted: 20 Dec, 2021 | Published: 27 Dec, 2021

Citation: Takahashi M, Bando Y, Fukui T, Maruyama A, Sugita M (2021) Effect of Clenching on Spinal Alignment in Normal Adults. Int J Dent Oral Health 8(1): dx.doi.org/10.16966/2378-7090.386

Copyright: ㄷ 2021 Takahashi M, et al. This is an open-access article distributed under the terms of the Creative Commons Attribution License, which permits unrestricted use, distribution, and reproduction in any medium, provided the original author and source are credited.

\section{Abstract}

Purpose: Occlusion plays a role in the execution of physical abilities in various. The aim of this study was to clarify the effect of clenching on the spinal alignment.

Materials and methods: Participants were 47 healthy men with no medical history involving the stomatognathic system and no musculoskeletal, neurological, or orthopedic problems. A spinal shape analyzer was used to measure spinal alignment. The Thoracic Kyphosis Angle (TKA), Lumbar Lordosis Angle (LLA), Sacral Slope Angle (SSA), and Spinal Inclination Angle (SIA) were measured in the sagittal plane. The measurement was performed while the participant was in a static standing posture and in a standing forward-bending posture. Measurements were performed under two conditions: while clenching and while relaxing. The occlusal balance was evaluated and divided into a stable group and an unstable group. For each posture, the differences in spinal alignment due to the presence or absence of clenching or the occlusal balance were analyzed using a splitplot design.

Results: No significant differences in spinal alignment due to occlusal balance were observed. In the static standing posture, there was no difference in the spinal alignment depending on clenching condition. However, in the standing forward-bending posture, LLA, SSA, and SIA values were significantly higher while relaxed than that while clenching.

Conclusion: As a result of this study, it was clarified that clenching influences the alignment of the spine during trunk flexion and restricts the flexion of the body, thereby contributing to the stability of the trunk.

Keywords: Clenching; Spinal alignment; Occlusal balance; Trunk stability

\section{Introduction}

Occlusion plays a role in the execution of physical abilities in various situations [1-6]. This is because occlusion affects the function of maintaining posture (postural control). Muscle activity in postural control involves mainly the antigravity muscles or trunk muscles [7]. The dorsal muscles (neck extensor muscles, erector spinae muscles, gluteus maximus, hamstrings, and triceps surae muscle) contribute more to postural control compared with the ventral muscles (neck flexor muscles, abdominal muscles, iliopsoas muscle, quadriceps femoris muscle, and tibialis anterior muscle). These muscles work in coordination with jaw and neck muscle activity associated with clenching and changes in jaw positions, thereby playing a role in the control of forward bending posture as well as increasing the sensitivity of somatosensory input $[8,9]$.

Occlusion influences somatosensory input and vestibular input, and several studies that examined the association between occlusion and postural control showed that body sway tended to be small when the occlusal contact status was favorable and that clenching with appropriate strength increased postural stability $[2,4,5,10,11]$. It has also been reported that occlusal contact status, use of mouthguards, and clenching influences physical ability and performance in sports that involve mainly agility, instantaneous force, muscle strength, and jumping power $[6,12-14]$. These studies focused on functional factors in the postural control function but have not been validated based on the morphological features of the body (e.g., spine, muscles, and joints). Meanwhile, the association between the morphological features of the body and physical ability in the context of postural control has been examined mainly in the field of physiotherapy [1519]. Although spinal alignment has been shown to influence the balance ability and gait speed and is also associated with the flexibility of the antigravity muscles, its association with occlusion has not yet been well studied.

The purpose of this study was to clarify the effect of occlusion on spinal alignment, which is a morphological factor in postural 
control. The null hypothesis was that spinal alignment is unaffected by clenching.

\section{Materials and Methods}

\section{Ethical approval of studies and informed consent}

This study was approved by the Ethics Committee of The Nippon Dental University School of Life Dentistry at Niigata (ECNG-R-443). The details of the study were described in full to all participants, and their informed consent was obtained written.

\section{Participants}

Participants were 47 healthy men (mean age; $23.0 \pm 1.2$ years) with no medical history involving the stomatognathic system and no musculoskeletal, neurological, or orthopedic problems. Exclusion criteria were tooth defects other than in the wisdom teeth, ongoing dental treatment, and presence of musculoskeletal pain or severe low back pain within the past 12 months, or a history of surgery in the lower limbs, spine, or pelvis.

\section{Measurement of spinal alignment}

A spinal shape analyzer (Spinal Mouse; Index Ltd., Tokyo, Japan) was used to measure spinal alignment $[20,21]$. The device transmits data to a computer via Bluetooth and the measured curvatures are shown on the computer display. The method has no medical risk or danger. The device has two rolling wheels that follow the spinous processes of the spine, and the measured distance and angles are transferred from the device to the computer. Data are sampled every $1.3 \mathrm{~mm}$ as the mouse is rolled along the spine, giving a sampling frequency of approximately $150 \mathrm{~Hz}$. This information is used by the manufacturer's software to calculate the relative positions of each vertebra, the angles between the vertebrae, and the total angle of the frontal and sagittal plane curvatures. The Thoracic Kyphosis Angle (TKA), Lumbar Lordosis Angle (LLA), Sacral Slope Angle (SSA), and Spinal Inclination Angle (SIA) were measured in the sagittal plane (Figure 1). The measurement was performed while the participant was in a static standing posture and in a standing forward-bending posture. TKA is the sum of the angles formed by the adjacent vertebral bodies from the $1^{\text {st }}$ thoracic vertebra to the $12^{\text {th }}$ thoracic vertebra. LLA is the sum of the angles formed by the adjacent vertebral bodies from

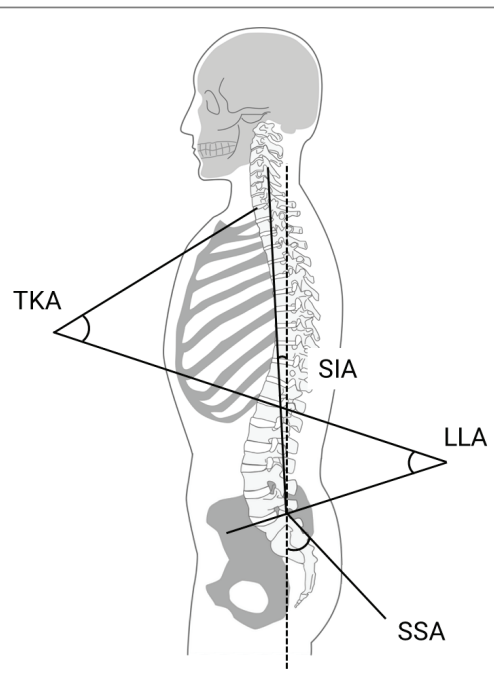

Figure 1: Spinal alignment in the sagittal plane.

TKA: Thoracic Kyphosis Angle, LLA: Lumbar Lordosis Angle, SSA: Sacral Slope Angle, SIA: Spinal Inclination Angle. the $12^{\text {th }}$ thoracic vertebra to the $1^{\text {st }}$ sacral vertebra. For TKA and LLA, a change in the kyphosis direction was defined as positive, and a change in the lordotic direction was defined as negative. SSA is the angle between the vertical line and the line connecting the $1^{\text {st }}$ and $3^{\text {rd }}$ sacral cords. SIA is the angle between the line connecting the $7^{\text {th }}$ cervical vertebra and the $1^{\text {st }}$ sacral vertebra and the vertical line. SSA and SIA were defined as positive for a forward lean and negative for a backward lean. Measurements were performed under two conditions: while clenching and while relaxing. While relaxing condition, participants were instructed with the mandibular resting position or do not touch the upper and lower teeth. One measurement was performed in about $5 \mathrm{sec}$, and the interval between measurements was $1 \mathrm{~min}$.

\section{Measurement of occlusal state}

The occlusal state was measured using a pressure-sensitive film (Dental Prescale, 50H-R type; Fujifilm Co., Ltd., Tokyo, Japan), and evaluated using an OCCLUZER (FPD-707; Fujifilm Co., Ltd.) $[6,13,22]$. In this study, participants with $<5 \%$ difference in the left and right occlusal contact area relative to the total occlusal contact area were assigned to the stable group and the all other participants were assigned to the unstable group. The stable and unstable groups were 21 and 26 participants, respectively.

\section{Statistical analysis}

Statistical analysis was performed in IBM SPSS 24.0 software (IBM Corp, Armonk, NY, USA). Significance was set at $\mathrm{P}<0.05$.

The reliability of the measured values under the clenching condition was examined. For the test of relative reliability, the intrarater reliability of random errors was tested by intraclass correlation coefficient (ICC) and decided to repeated measurements by decision study. For the test of absolute reliability, the detection of systematic errors was performed by Bland-Altman analysis.

Next, the left-right differences in occlusal contact area between the stable group and the unstable group were compared. Because there was a level where normality was not recognized by the Shapiro-Wilk test, the median percentage of the high value side with respect to the total occlusal contact area was compared using the Mann-Whitney U test.

Finally, for the static standing posture and the standing forwardbending posture, the differences in spinal alignment due to the presence or absence of clenching ortheocclusal balance was analyzed using a split-plot design. The factors that showed a significant difference were compared using a paired t-test because normality was observed at each level.

\section{Results}

Table 1 shows the test results for relative and absolute reliability. The results of the Bland-Altman analysis of evaluation data showed no fixed or proportional biases. All ICC $(1,1)$ showed high values, and the results of the decision study indicated that two repeated measurements were required to obtain a confidence coefficient of 0.9 or higher.

Figure 2 shows a comparison of the median percentages on the high side of the total occlusal contact area. The laterality of the occlusal contact area was significantly smaller in the stable group than in the unstable group $(\mathrm{P}<0.01)$.

Table 2 and figure 3 shows the test results of the split-plot design according to the clenching condition and the occlusal balance of spinal alignment in the static standing posture. No significant differences were observed in the factors according to clenching condition or occlusal balance in any of the spinal alignments. 
Table 1: Relative and absolute reliability of spinal alignment. Thoracic Kyphosis Angle (TKA), Lumbar Lordosis Angle (LLA), Sacral Slope Angle (SSA), and Spinal Inclination Angle (SIA) were measured using the Spinal Mouse. A: static standing posture, B: standing bending-forward posture.

A

\begin{tabular}{|c|c|c|c|c|c|c|c|c|c|}
\hline & & & & & & \multicolumn{4}{|c|}{ Bland-Altman analysis } \\
\hline & & & & & & \multirow{2}{*}{\multicolumn{2}{|c|}{$\begin{array}{c}\text { Fixed bias } \\
95 \% \mathrm{Cl}\end{array}$}} & \multirow{2}{*}{\multicolumn{2}{|c|}{$\begin{array}{l}\text { Proportional bias } \\
\text { Uncorrelated test }\end{array}$}} \\
\hline & $\operatorname{ICC}(1,1)$ & \multicolumn{2}{|c|}{ 95\% Cl (degree) } & \multirow{2}{*}{ P-Value } & \multirow{2}{*}{$\mathrm{MDC}_{95}$ (degree) } & & & & \\
\hline & & Lower limit value & Upper limit value & & & Lower limit value & Upper limit value & $\mathbf{r}$ & P-Value \\
\hline TKA & 0.849 & 0.731 & 0.916 & $<0.001$ & 2.99 & -0.30 & 1.70 & 0.163 & 0.275 \\
\hline LLA & 0.882 & 0.789 & 0.934 & $<0.001$ & 3.76 & -1.10 & 2.12 & 0.164 & 0.272 \\
\hline SSA & 0.847 & 0.726 & 0.915 & $<0.001$ & 2.95 & -1.48 & 0.67 & 0.161 & 0.280 \\
\hline SIA & 0.842 & 0.718 & 0.912 & $<0.001$ & 1.65 & -0.36 & 0.78 & 0.004 & 0.978 \\
\hline
\end{tabular}

ICC (1,1): Intra-rater Reliability

$95 \% \mathrm{Cl}: 95 \%$ Confidence Interval

$\mathrm{MDC}_{95}: 95 \%$ Minimal Detectable Change

B

\begin{tabular}{|c|c|c|c|c|c|c|c|c|c|}
\hline & & & & & & \multicolumn{4}{|c|}{ Bland-Altman analysis } \\
\hline & & & & & & \multirow{2}{*}{\multicolumn{2}{|c|}{$\begin{array}{c}\text { Fixed bias } \\
95 \% \mathrm{Cl}\end{array}$}} & \multirow{2}{*}{\multicolumn{2}{|c|}{$\begin{array}{l}\text { Proportional bias } \\
\text { Uncorrelated tes }\end{array}$}} \\
\hline & $\operatorname{ICC}(1,1)$ & \multicolumn{2}{|c|}{ 95\% Cl (degree) } & \multirow{2}{*}{ P-Value } & \multirow{2}{*}{$\mathrm{MDC}_{95}$ (degree) } & & & & \\
\hline & & Lower limit value & Upper limit value & & & Lower limit value & Upper limit value & $r$ & P-Value \\
\hline TKA & 0.878 & 0.791 & 0.930 & $<0.001$ & 3.19 & -1.16 & 1.55 & 0.228 & 0.122 \\
\hline LLA & 0.913 & 0.850 & 0.951 & $<0.001$ & 3.93 & -2.50 & 0.33 & 0.208 & 0.160 \\
\hline SSA & 0.889 & 0.826 & 0.942 & $<0.001$ & 3.01 & -2.26 & 0.39 & 0.219 & 0.139 \\
\hline SIA & 0.969 & 0.946 & 0.983 & $<0.001$ & 2.38 & -2.53 & 0.31 & 0.070 & 0.642 \\
\hline
\end{tabular}

ICC (1,1): Intra-rater Reliability

95\% Cl: 95\% Confidence Interval

$\mathrm{MDC}_{95}: 95 \%$ Minimal Detectable Change

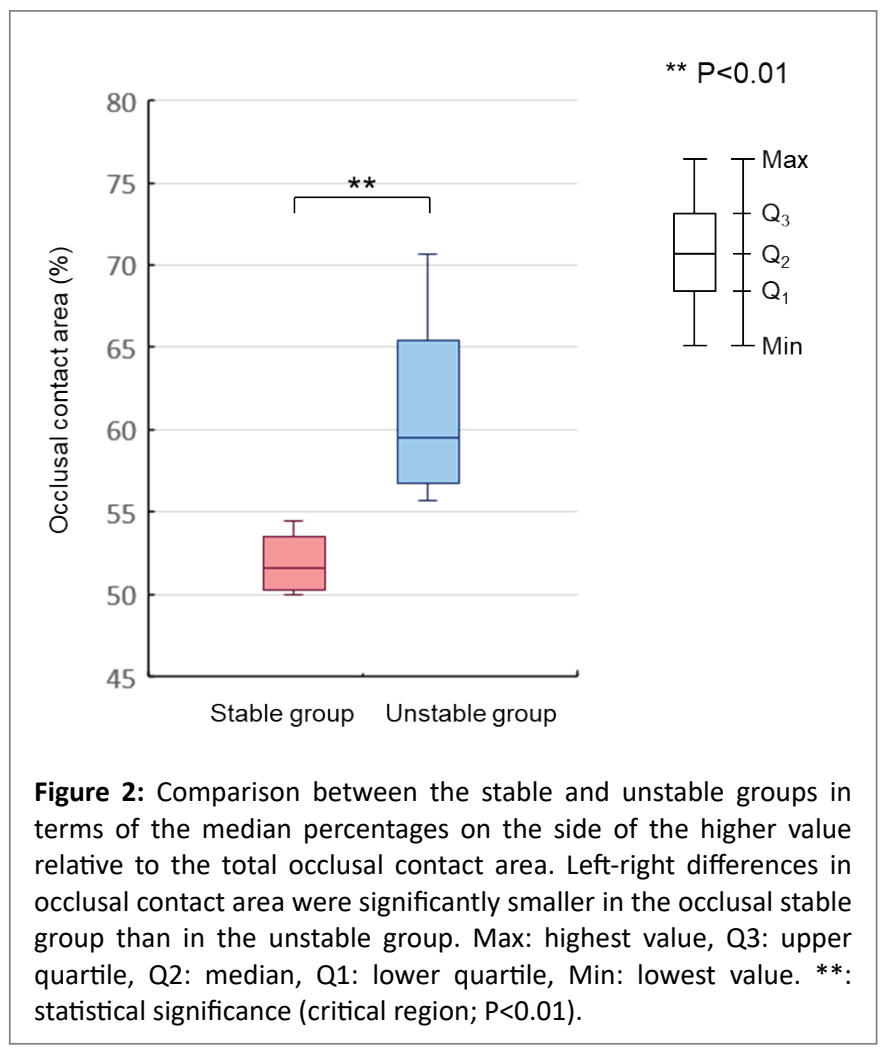

Table 3 and figure 4 show the test results of the split-plot design based on the clenching condition and the occlusal balance of spinal alignment in the standing forward-bending posture. Differences while clenching and while relaxed were observed in LLA, SSA, and SIA, and the values were higher while relaxed. In addition, the significant differences between levels were all larger than those of $\mathrm{MDC}_{95}$ and were judged to be true changes.

\section{Discussion and Conclusion}

The results of this study showed that spinal alignment is affected by clenching. Therefore, the null hypothesis was rejected.

When investigating the influence of clenching on spinal alignment, differences in the occlusal contact status among participants might influence the measurements in the clenching condition. This is because, favorable occlusal balance or evenness of occlusal contact achieved by wearing a mouthguard affects postural control [6,12-14]. Thus, this study divided participants into a stable group and an unstable group depending on the differences in occlusal contact area between the right and left sides, confirmed that there were significant differences in the occlusal contact status between the groups, and investigated the influence of clenching on spinal alignment.

In the static standing posture, there were no significant differences in spinal alignment between the clenching and relaxed conditions or by occlusal balance status. Spinal curvature tends to be established with the development of standing postural control and can be modified by environmental factors (e.g., posture while working and 
Table 2: Results of the split-plot design in the static standing posture. A: Thoracic Kyphosis Angle (TKA), B: Lumbar Lordosis Angle (LLA), C: Sacral Slope Angle (SSA), D: Spinal Inclination Angle (SIA).

A

\begin{tabular}{|l|r|r|r|r|r|}
\hline Source & SS & $\mathbf{d f}$ & MS & F value & P value \\
\hline Clenching or relaxing condition \\
\hline A & 1.517 & 1 & 1.517 & 0.155 & 0.696 \\
\hline Error (A) & 440.228 & 45 & 9.783 & & \\
\hline Occlusal balance \\
\hline B & 65.179 & 1 & 65.179 & 0.962 & 0.332 \\
\hline A $\times$ B & 3.687 & 1 & 3.687 & 0.377 & 0.542 \\
\hline Error (B) & 3049.034 & 45 & 67.756 & & \\
\hline
\end{tabular}

SS: Sum of Squares; df: Degree of Freedom; MS: Mean Square

B

\begin{tabular}{|c|c|c|c|c|c|}
\hline Source & SS & df & MS & F value & $P$ value \\
\hline \multicolumn{6}{|c|}{ Clenching or relaxing condition } \\
\hline A & 6.600 & 1 & 6.600 & 0.853 & 0.361 \\
\hline Error (A) & 348.209 & 45 & 7.738 & & \\
\hline \multicolumn{6}{|c|}{ Occlusal balance } \\
\hline B & 0.329 & 1 & 0.329 & 0.006 & 0.937 \\
\hline$A \times B$ & 3.536 & 1 & 3.536 & 0.457 & 0.503 \\
\hline Error (B) & 2322.905 & 45 & 51.620 & & \\
\hline
\end{tabular}

SS: Sum of Squares; df: Degree of Freedom; MS: Mean Square

C

\begin{tabular}{|c|c|c|c|c|c|}
\hline Source & SS & df & MS & F value & $P$ value \\
\hline \multicolumn{6}{|c|}{ Clenching or relaxing condition } \\
\hline A & 3.228 & 1 & 3.228 & 0.422 & 0.519 \\
\hline Error (A) & 344.177 & 45 & 7.648 & & \\
\hline \multicolumn{6}{|c|}{ Occlusal balance } \\
\hline B & 5.168 & 1 & 5.168 & 0.290 & 0.593 \\
\hline$A \times B$ & 0.249 & 1 & 0.249 & 0.033 & 0.858 \\
\hline Error (B) & 801.386 & 45 & 17.809 & & \\
\hline
\end{tabular}

SS: Sum of Squares; df: Degree of Freedom; MS: Mean Square

D

\begin{tabular}{|c|c|c|c|c|c|}
\hline Source & SS & df & MS & F value & $P$ value \\
\hline \multicolumn{6}{|c|}{ Clenching or relaxing condition } \\
\hline A & 0.025 & 1 & 0.025 & 0.019 & 0.891 \\
\hline Error (A) & 60.209 & 45 & 1.338 & & \\
\hline \multicolumn{6}{|c|}{ Occlusal balance } \\
\hline B & 10.045 & 1 & 10.045 & 1.941 & 0.170 \\
\hline$A \times B$ & 0.281 & 1 & 0.281 & 0.210 & 0.649 \\
\hline Error (B) & 232.828 & 45 & 5.174 & & \\
\hline
\end{tabular}

SS: Sum of Squares; df: Degree of Freedom; MS: Mean Square lifestyle) $[23,24]$. In the relaxed condition, all participants showed typical spinal alignment, indicating that the healthy adult population selected as participants in this study represented the standard population. Conscious postural control is not required to maintain the quiet standing posture, but fascial tension contributes to it [25]. The activity of antigravity muscles is low [26]. For these reasons, muscles that function upon clenching might not have a large effect on postural control and thus no effect on spinal alignment.

Meanwhile, it has been reported that there are differences between the sexes in the characteristics of spinal movement when bending the trunk forward, and this can have an effect on dorsal abdominal muscle activity $[27,28]$. It has also been reported that antigravity muscles work to control the standing posture with respect to the forward leaning posture and these muscles increase the sensitivity of somatosensory input $[8,9]$. Clenching reinforces the skeletal muscles of the limbs in order to stabilize the direction of the body [29]. The masseter muscle belonging to the Deep Front Line (DFL) and the sternocleidomastoid muscle belonging to the Lateral Line (LTL), Superficial Front Line (SFL), and Superficial Back Line (SBL) contribute to the stabilization of the body [30,31]. Favorable occlusal balance supports the stabilization of the trunk, and thus, clenching is advantageous for movements that involve mainly the retention of the trunk $[6,13]$. However, the stabilization of the trunk by muscles and fasciae does not have a favorable effect during movements that require trunk flexion. Also, given that the sternocleidomastoid muscle is involved in orienting the head in relation to the trunk and head movements, and its activity positively correlates with the force applied on teeth [32-34], clenching may influence the muscles and fasciae in the LTL, SFL, and SBL. Taken together, occlusal state (clenching/relaxing and occlusal balance) is likely to influence the curvature and the range of motion of the spine. Among the muscles associated with the spine, deep trunk muscles with insertion and origin at the spine are involved in the control of lumbar curvature and mechanical stability between the vertebral bodies [35]. Because these muscles belonging to the DFL, the actions of the local system [35] may be influenced by occlusion. Also, the stability of the head is supported by masticatory muscles, neck muscles, and shoulder muscles, which act as antigravity muscles, and the neck muscles work in coordination with the abdominal muscles [26,32]. These facts support the association between the SFL and trunk stability [30,31]. This study showed significantly low LLA, SSA, and SIA in the standing forward-bending posture in the clenching condition. This is likely due to clenching-induced activity of the abdominal muscles that connect to the DFL and SFL as well as the activity of dorsal muscles in the SBL that contribute to trunk stability. Occlusal balance has a positive effect on the physical abilities exerted by the stabilization of the trunk [6]. However, trunk flexion is not achieved by contracting or tightening muscles and fasciae but is rather by relaxing them. This may explain why occlusal balance did not have an impact on spinal alignment in the standing forward-bending posture. Each spinal alignment component showed different results, and this can be considered according to the anatomical features of the body. Movement of the thoracic spine is restricted by the costovertebral joints and sternocostal joints adjacent to the thoracic intervertebral joint [33], and thus, the influence of the muscles and fasciae associated with occlusion on thoracic curvature is likely to be small. In contrast, in the lumbar spine, the joint components are largely restricted in the rotation and lateral flexion of the trunk, but those are less restricted in the flexion and extension movements and are easily affected by muscles [33]. This may explain why clenching did not influence the thoracic curvature but did influence the lumbar curvature through muscles and fasciae associated with clenching. Also, SSA reflects the pelvic tilt, which corresponds to the hip joints 
A

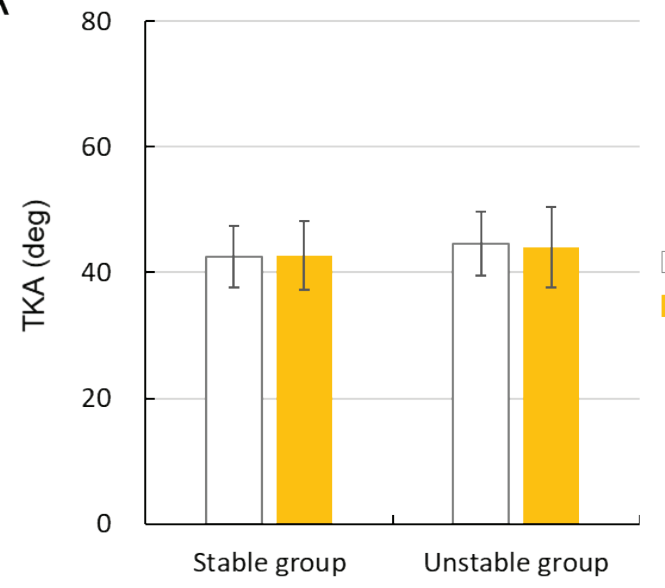

B

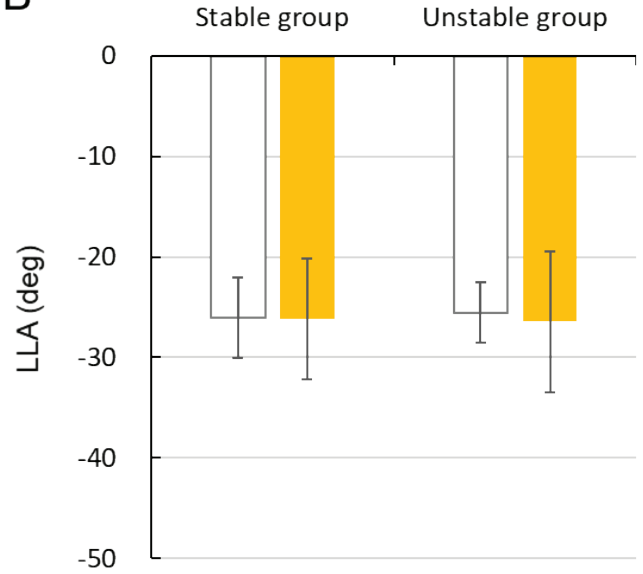

C

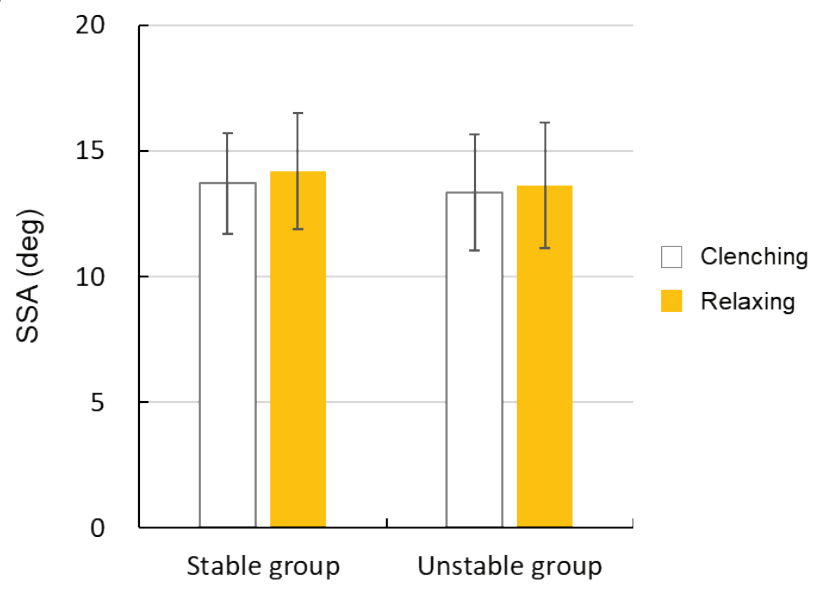

D

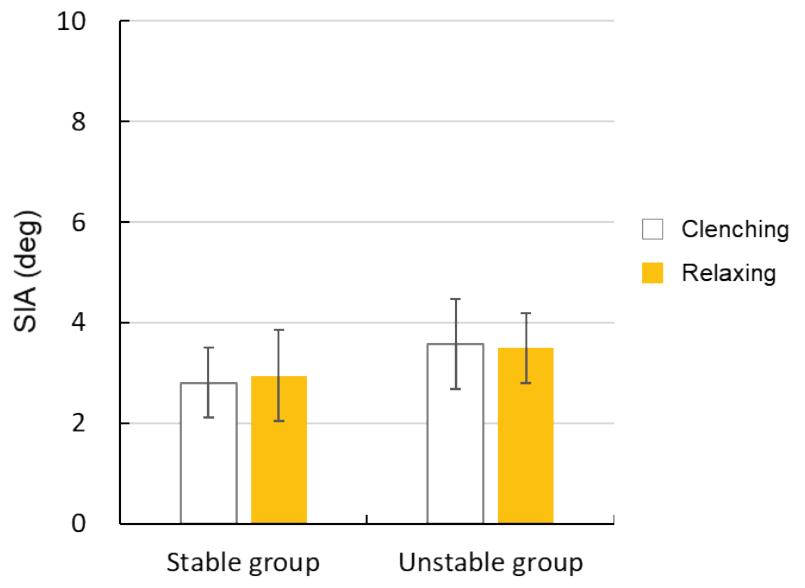

Figure 3: Differences in spinal alignment due to occlusion in the static standing posture. Measurements are expressed as means \pm SD. Error bar indicates standard error of the mean. No significant differences were observed between the clenching and relaxing condition in any spinal alignment. In addition, no significant differences were observed between the stable and unstable groups. A: Thoracic Kyphosis Angle (TKA), B: Lumbar Lordosis Angle (LLA), C: Sacral Slope Angle (SSA), D: Spinal Inclination Angle (SIA).

Table 3: Results of the split-plot design in the standing bending-forward posture. A: Thoracic Kyphosis Angle (TKA), B: Lumbar Lordosis Angle (LLA), C: Sacral Slope Angle (SSA), D: Spinal Inclination Angle (SIA).

A

\begin{tabular}{|c|c|c|c|c|c|}
\hline Source & SS & df & MS & F value & $P$ value \\
\hline \multicolumn{6}{|c|}{ Clenching or relaxing condition } \\
\hline A & 9.905 & 1 & 9.905 & 0.518 & 0.476 \\
\hline Error (A) & 860.946 & 45 & 19.132 & & \\
\hline \multicolumn{6}{|c|}{ Occlusal balance } \\
\hline B & 18.981 & 1 & 18.981 & 0.116 & 0.735 \\
\hline$A \times B$ & 6.714 & 1 & 6.714 & 0.351 & 0.557 \\
\hline Error (B) & 7373.019 & 45 & 163.845 & & \\
\hline
\end{tabular}

SS: Sum of Squares; df: Degree of Freedom; MS: Mean Square

\section{B}

\begin{tabular}{|l|r|r|r|r|r|}
\hline Source & SS & df & MS & F value & P value \\
\hline Clenching or relaxing condition \\
\hline A & 667.477 & 1 & 667.477 & 49.042 & $<0.001^{* *}$ \\
\hline Error (A) & 612.459 & 45 & 13.610 & & \\
\hline Occlusal balance \\
\hline B & 30.007 & 1 & 30.007 & 0.235 & 0.630 \\
\hline A × B & 34.414 & 1 & 34.414 & 2.529 & 0.119 \\
\hline Error (B) & 5749.418 & 45 & 127.765 & & \\
\hline
\end{tabular}

SS: Sum of Squares; df: Degree of Freedom; MS: Mean Square $* * \mathrm{P}<0.01$ : denotes statistical significance 
C

\begin{tabular}{|l|r|r|r|r|r|}
\hline Source & SS & df & MS & F value & P value \\
\hline Clenching or relaxing condition \\
\hline A & 129.995 & 1 & 129.995 & 9.565 & $0.003^{* *}$ \\
\hline Error (A) & 611.558 & 45 & 13.590 & & \\
\hline Occlusal balance \\
\hline B & 21.934 & 1 & 21.934 & 0.228 & 0.635 \\
\hline A $\times$ B & 3.102 & 1 & 3.102 & 0.228 & 0.635 \\
\hline Error (B) & 4324.386 & 45 & 96.097 & & \\
\hline
\end{tabular}

SS: Sum of Squares; df: Degree of Freedom; MS: Mean Square $* * p<0.01$ : denotes statistical significance
D

\begin{tabular}{|l|r|r|r|r|r|}
\hline Source & SS & df & MS & F value & P value \\
\hline Clenching or relaxing condition \\
\hline A & 630.375 & 1 & 630.375 & 25.164 & $<0.001 * *$ \\
\hline Error (A) & 1127.264 & 45 & 25.050 & & \\
\hline Occlusal balance \\
\hline B & 110.754 & 1 & 110.754 & 0.192 & 0.663 \\
\hline A $\times$ B & 3.055 & 1 & 3.055 & 0.122 & 0.729 \\
\hline Error (B) & 23611.055 & 45 & 524.690 & & \\
\hline
\end{tabular}

SS: Sum of Squares; df: Degree of Freedom; MS: Mean Square

${ }^{* *} \mathrm{P}<0.01$ : denotes statistical significance
A

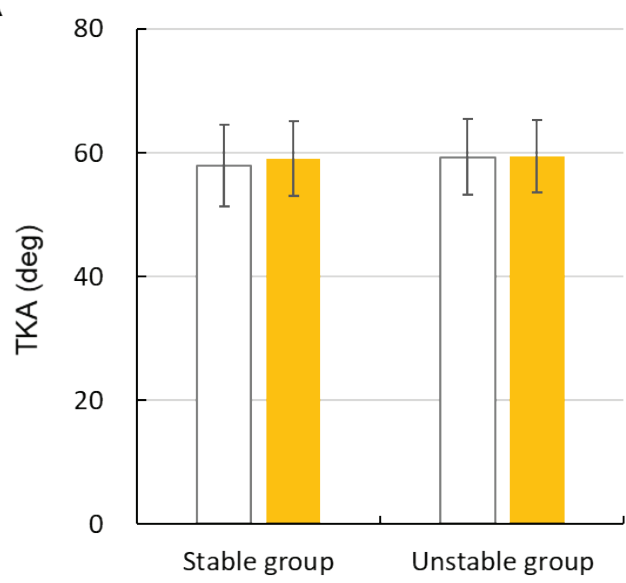

B

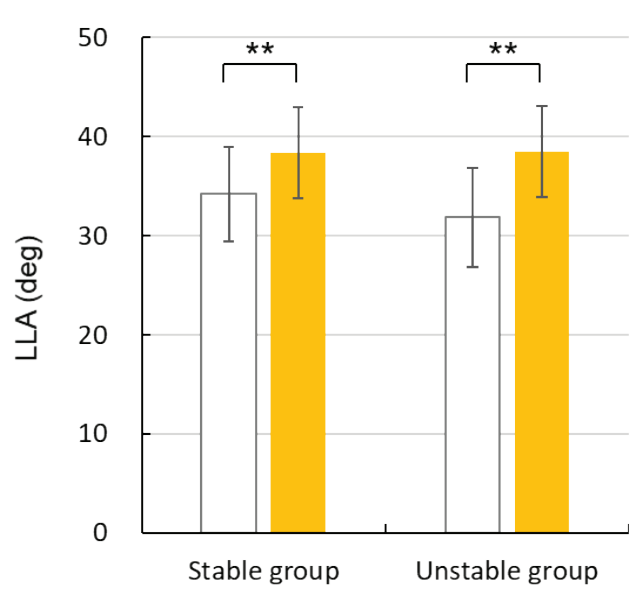

C

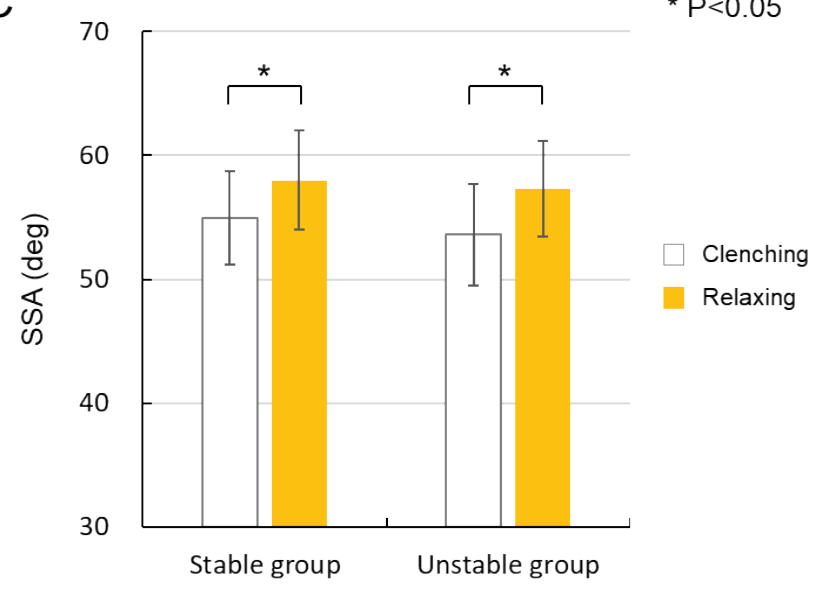

D

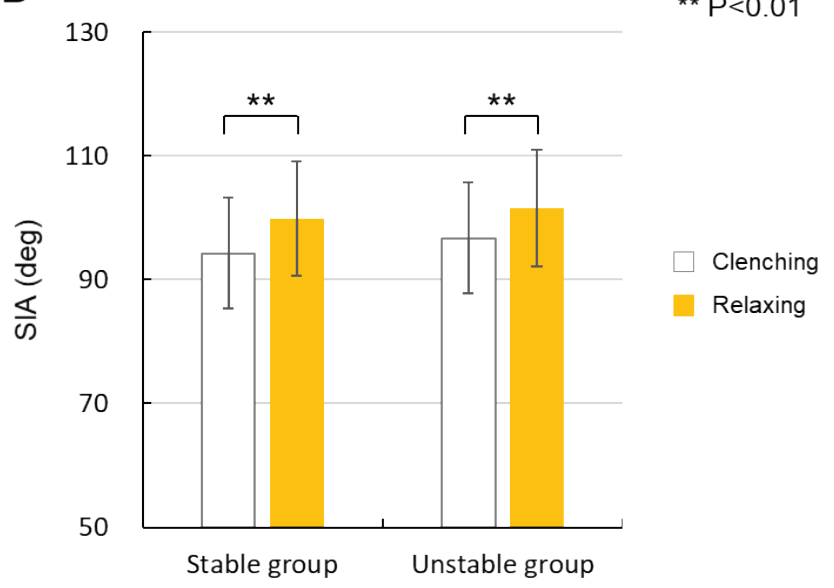

Figure 4: Differences in spinal alignment due to occlusion in the standing bending-forward posture. Measurements are expressed as means \pm SD. Error bar indicates standard error of the mean. ${ }^{* *},{ }^{*}$ : statistical significance (critical region; ${ }^{*} \mathrm{P}<0.01,{ }^{*} \mathrm{P}<0.05$ ). In LLA, SSA, and SIA, the relaxing condition showed significantly higher values compared with clenching condition. The same tendency was observed in the stable and unstable groups. However, no significant differences were observed between the stable and unstable groups. A: Thoracic Kyphosis Angle (TKA), B: Lumbar Lordosis Angle (LLA), C: Sacral Slope Angle (SSA), D: Spinal Inclination Angle (SIA). 
that move with the pelvis in a coordinated manner when the knee joints are extended. The muscles and fasciae surrounding the sacrum are in the SBL, which is used to maintain the standing posture and to restrict trunk flexion [30,31]. In this study, SSA was lower in the clenching condition, suggesting that clenching might have contributed to the restriction of pelvic tilt and hip flexion when the trunk was flexing. SIA was also lower in the clenching condition. Taken together, these findings suggest that clenching influences the alignment of the spine during trunk flexion and restricts the flexion of the body, thereby contributing to the stability of the trunk.

This study has several limitations. First, the participants were limited to healthy men, and thus, differences according to sex, age, and disease status were not examined. In addition, differences according to clenching intensity were not investigated. Differences in spinal movement during forward bending of the trunk between men and women [27] as well as the influence of antigravity muscle activity [28] have been reported, and these factors need to be investigated in the future.

\section{Data Availability}

The datasets collected and/or analyzed during the current study are available from the corresponding author on reasonable request.

\section{Acknowledgments}

This work was supported by JSPS KAKENHI Grant Number JP18K09668 and the Nippon Dental University Intramural Research Fund (NDU Grants N-21004).

\section{Conflicts of Interest Statement}

The authors have no conflicts of interest relevant to this article.

\section{References}

1. Williams MO, Chaconas SJ, Bader P (1983) The effect of mandibular position on appendage muscle strength. J Prosthet Dent 49: 560567.

2. Bracco P, Deregibus A, Piscetta R, Ferrario G (1998) Observations on the correlation between posture and jaw position: a pilot study. Cranio 16: 252-258.

3. Valentino B, Melito F, Aldi B, Valentino T (2002) Correlation between interdental occlusal plane and plantar arches: An EMG study. Bull Group Rech Sci Stomatol Odontol 44: 10-13.

4. Bracco P, Deregibus A, Piscetta R (2004) Effects of different jaw relations on postural stability in human subjects. Neurosci Lett 356 : 228-230.

5. Alghadir AH, Zafar H, Iqbal ZA (2015) Effect of three different jaw positions on postural stability during standing. Funct Neurol 30: 5357.

6. Takahashi M, Bando Y (2018) Relationship between occlusal balance and agility in Japanese elite female junior badminton players. Int J Sports Dent 11: 34-42.

7. Ghamkhar L, Kahlaee AH (2019) The effect of trunk muscle fatigue on postural control of upright stance: A systematic review. Gait Posture 72: 167-174.

8. Fitzpatrick R, McCloskey DI (1994) Proprioceptive, visual and vestibular thresholds for the perception of sway during standing in humans. J Physiol 478: 173-186.

9. Gatev P, Thomas S, Kepple T, Hallett M (1999) Feedforward ankle strategy of balance during quiet stance in adults. J Physiol 514: 915928.
10. Eunse P, Gregor S, John PS (2012) Functional synergies underlying control of upright posture during changes in head orientation. PLOS One 7: e41583.

11. Gangloff P, Louis JP, Perrin PP (2000) Dental occlusion modifies gaze and posture stabilization in human subjects. Neuroscience Letters 293: 203-206.

12. Bernat B, Daniel MD, Javier P, Jose M, Mònica ST, et al. (2018) Effects of jaw clenching wearing customized mouthguards on agility, power and vertical jump in male high-standard basketball players. J Exerc Sci Fit 16: 5-11.

13. Takahashi M, Bando Y, Kitaoka K, Kimura S (2020) Influence of occlusal state on posture control and physical fitness of elite athletes: Examination targeting female handball players. J Sports Dent 24: 18-25.

14. Adrià $M$, Bernat $B$, Joan $A C$, Jordi AA (2021) Acute effects of wearing bite-aligning mouthguards on muscular strength, power, agility and quickness in a trained population: a systematic review. Int J Environ Res Public Health 18: 6933.

15. Phelps WM, Kiphuth RJH, Goff CW (1956) The diagnosis and treatment of postural defects. $2^{\text {nd }}$ edition. Charles $C$ Thomas: Springfield III, p.41-43.

16. Mehrsheed S, Robert HB, Christine AH, Dirk RL, Kenton RK (2005) Balance disorder and increased risk of falls in osteoporosis and kyphosis: significance of kyphotic posture and muscle strength. Osteoporos Int 16: 1004-1010.

17. Imagama S, Ito Z, Wakao N, Seki T, Hirano K, et al. (2013) Influence of spinal sagittal alignment, body balance, muscle strength, and physical ability on falling of middle-aged and elderly males. Eur Spine J 22: 1346-1353.

18. Hasebe K, Sairyo K, Hada Y, Dezawa A, Okubo Y, et al. (2014) Spinopelvic-rhythm with forward trunk bending in normal subjects without low back pain. Eur J Orthop Surg Traumatol 24: S193-S199.

19. Sadler SG, Spink MJ, Ho A, De Jonge XJ, Chuter VH (2017) Restriction in lateral bending range of motion, lumbar lordosis, and hamstring flexibility predicts the development of low back pain: a systematic review of prospective cohort studies. BMC Musculoskelet Disord 18: 179.

20. Mannion AF, Knecht K, Balaban G, Dvorak J, Grob D (2004) A new skin-surface device for measuring the curvature and global and segmental ranges of motion of the spine: reliability of measurements and comparison with data reviewed from the literature. Eur Spine J 13: 122-136.

21. Post RB, Leferink VJM (2004) Spinal mobility: sagittal range of motion measured with the Spinal Mouse, a new non-invasive device. Arch Othop Trauma Surg124: 187-192.

22. Takahashi M, Takahashi F, and Morita $O$ (2008) Evaluation of the masticatory part and the habitual chewing side by wax cube and bite force measuring system (Dental Prescale). J Jpn Prosthodont 52: 513-520.

23. Frank S, Virginie L, Reid B, Wasa S, Jean-Pierre F (2006) Gravity line analysis in adult volunteers: age-related correlation with spinal parameters, pelvic parameters, and foot position. Spine (Phila $\mathrm{Pa}$ 1976) 31: E959-E967.

24. Okuni I, Uchi M, Harada T (2006) Sagittal-plane spinal curvature and center of foot pressure in healthy young adults. J Med Soc Toho 53: 254-260.

25. Stecco L (2004) Fascial manipulation for musculoskeletal pain. Padova, Piccin, p.12-20,41-49,88,111-121. 
26. Mitani Y, Morikita I (2008) Correlation between sagittal plane alignment of the lumbosacral spine and trunk muscle strength and leg muscle flexibility in a static, upright position. Rigakuryoho Kagaku 23: 35-38.

27. Scholtes SA, Van Dillen LR (2007) Gender related differences in prevalence of lumbopelvic region movement impairments in people with low back pain. J Orthop Sports Phys Ther 37: 744-753.

28. Comerford M, Mottram S (2017) Kinetic control the management of uncontrolled movement. Tokyo: Book House HD, p.43-49.

29. Boroojerdi B, Battaglia F, Muellbacher W, Cohen LG (2000) Voluntary teeth clenching facilitates human motor system excitability. Clin Neurophysiol 111: 988-993.

30. Thomas WM (2016) Anatomy trains. $3^{\text {rd }}$ edition, Tokyo: Igaku-shoin, p.81-146, 201-227.
31. Robert S, Amanda B (2019) Fascia in sport and movement. Tokyo: Round flat, p.47-62.

32. Davis PL (1979) Electromyographic study of superficial neck muscles in mandibular function. J Dent Res 58:537-538.

33. Kapandji IA (1986) Capandy Physiologie articulaire III. Tokyo: Ishiyaku, p.28-29,212.

34. Kohno S, Yoshida K, Kobayashi H, Miura H (1987) The EMG activities of the sternocleidomastoid muscle during occlusal function. Nihon Hotetsu Shika Gakkai Zasshi 31: 764-769.

35. Bergmark A (1989) Stability of the lumbar spine: a study in mechanical engineering. Acta Orthop Scand Suppl 230: 1-54. 